\title{
ON AN ASYMPTOTIC FORMULA FOR THE NIVEN NUMBERS
}

\section{CURTIS N. COOPER and ROBERT E. KENNEDY}

Department of Mathematics and Computer Science

Certral Missouri State University

Warrenshure, Missouri 64093 U.S.A.

(Received May 20, 1985)

ABSTRACT. A Niven number is a positive integer which is divisible by its digital sum. 4 discussion of the possibility of an asymptotic formula for $\mathrm{N}(\mathrm{x})$ is given. Here, $N(x)$ denotes the number of Niven numbers less than $x$. A partial result will be pressented. This result will be an asymptotic formula for $\mathrm{N}_{k}(x)$ which denotes the number of Niven numbers less than $x$ with digital sum $k$.

NTY WORDS AND PHRASES. Digital sums, asymptotic formula, Niven number. 1980 MATHEMATICS SUBJECT CLASSIFICATION CODE, $10 \mathrm{H} 25,10 \mathrm{~A} 30$

1. INTRODUCTION.

In Kennedy et al [1] the concept of a Niven number was introduced as any positive integer $n$ which is divisible by its digital sum $s(n)$. One of the first questions about the set, $N$, of Niven numbers which was investigated was the status of

$$
\operatorname{Lim}_{x \rightarrow \infty} \frac{N(x)}{x}
$$

where $N(x)$ denotes the number of Niven numbers less than $x$. (In what follows, we will use the convention that if $A$ is a set of integers, then $A(x)$ will be the number of members of $A$ less than $x_{\text {.) }}$ This limit, if it exists, is called the "natural density" of the set $N$.

Even though this was answered in Kennedy and Cooper [2], (the natural density of $N$ is zero), other questions demanded attention. In particular, "Can an asymptotic formula for $\mathrm{N}(\mathrm{x})$ be determined?" That is, does there exist a function $f(x)$ such that

$$
\operatorname{Lim}_{x \rightarrow \infty} \frac{N(x)}{f(x)}=1 ?
$$

If such an $f(x)$ exists, then the usual notation to indicate this is,

$$
\mathrm{N}(\mathrm{x}) \sim \mathrm{f}(\mathrm{x})
$$

The following notation will be used to arrive at a partial answer to this question. I.et $k$ be a positive integer. Then $k$ may be written in the form

$$
k=2^{a} 5^{b} t
$$


where $(t, 10)=1$. We define:

$$
\begin{aligned}
& s_{k}=\{x: s(x)=k\} . \\
& r_{k}=S_{k} \cap N, \\
& \left.g_{k}\right)=\max \{a, b\},
\end{aligned}
$$

and

$$
e(k)=\text { order of } 10 \text { modulo } t \text {. }
$$

In what follows, we will develop an asymptotic formula for $N_{k}(x)$.

2. AN ASYMPTCTIC FORNUIA WHEN $k=2^{a} 5^{b} 3^{c}$.

Simh a formula for $N_{k}(x)$ can easily be found for $k$ of the form $2^{a} 5^{b} 3^{c}$ when $c=0,1$, or 2 . This is given in Theoren 2.1 with the help of the following lemma.

IIM 1. Tet $k, n$ bo integers. Then

$$
\left(\begin{array}{c}
{\left[\frac{n-\bar{e}(x)-1}{e(k)}\right]+1} \\
k
\end{array}\right) \leq N_{k}\left(10^{n}\right)
$$

PROCF. Here, the "are brackets denote the greatest. integer function and the parewthese lanste a binomial coefficient. Note that an integer of the fort:

$$
\sum_{i=0}^{f} e_{1} \overline{e(k)+i e(k)}
$$

where $c_{i} \in\{0,1\}, c_{0}+c_{1}+c_{2}+\ldots+c_{f}=k$, and

$$
f=\left[\frac{n-\bar{e}(k)-1}{e(k)}\right]
$$

is a Niven number with digital sum $k$. But the sequence

$$
\left\{c_{i}\right\}_{i=0}^{f}
$$

can be rearranged exactly

$$
\left(\begin{array}{c}
f+1 \\
k
\end{array}\right)
$$

ways, and each of these will determine a Niven number with digital sum $k$. The refore, we have that (2.1) holds.

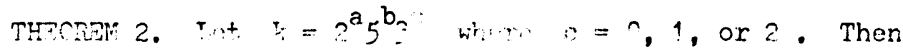

$$
\because(x) \sim(\log x)^{k} / k !
$$

PROOF. Let $n$ be the positive integer such that

$$
10^{\mathrm{n}} \leqslant x<10^{\mathrm{n}+1}
$$

For $k$ of the civen form, $e(k)=1$, and it follows frot Tand [?] that 


$$
\mathrm{s}_{\mathrm{k}}\left(10^{\mathrm{n}}\right) \sim\left(\begin{array}{c}
\mathrm{n}-\mathrm{m} \\
\mathrm{k}
\end{array}\right) \sim \mathrm{n}^{\mathrm{k}} / \mathrm{k} \text { ! }
$$

for $m$ not dependent on $n$. By Iemma 1 , we have

$$
\left(\begin{array}{c}
n-\bar{e}(k) \\
k
\end{array}\right) \leqslant N_{k}\left(10^{n}\right) \leqslant S_{k}\left(10^{n}\right)
$$

and so,

$$
\mathrm{N}_{\mathrm{k}}\left(10^{\mathrm{n}}\right) \sim \mathrm{n}^{\mathrm{k}} / \mathrm{k} !
$$

since each side of $(2.10)$ is asymptotic to $\mathrm{n}^{\mathrm{k}} / \mathrm{k}$ ! . From

$$
N_{k}\left(10^{n}\right) \leqslant N_{k}(x) \leq N_{k}\left(10^{n+1}\right)
$$

and

$$
\mathrm{N}_{\mathrm{k}}\left(10^{n}\right) \sim \mathrm{n}^{\mathrm{k}} / \mathrm{k} ! \sim(\mathrm{n}+1)^{\mathrm{k}} / \mathrm{k} ! \sim \mathrm{N}_{\mathrm{k}}\left(10^{\mathrm{n}+1}\right)
$$

it readily follows that

$$
v_{s}(x) \sim(\log x)^{k} / k !
$$

because $n=[\log x] \sim \log x$.

3. A LOWER BOIND FOR $\mathrm{H}(\mathrm{x})$.

It should be noted here that Lemma 1 can be used to determine a lower bound for $N(x)$. In fact, the search for such a lower bound led to the method that will give us an asymptotic formula for $\mathrm{N}_{k}(x)$. To determine this lower bound, let $k=2^{m}$ for some positive integer $m$. Then $e(k)=1$ and $\bar{e}(k)=m$, and we have by Lemma 1 that

Thus,

$$
\left(\begin{array}{c}
n-m \\
2^{m}
\end{array}\right) \leqslant N_{2^{m}\left(10^{n}\right)}
$$

$$
N\left(10^{n}\right) \geqslant N{ }_{2^{m}}\left(10^{n}\right) \geqslant\left(\begin{array}{c}
n-m \\
2^{m}
\end{array}\right) \geqslant n^{2^{m}-1}
$$

eventually. That is, there exists an integer $K$ such that

$$
N\left(10^{n}\right) \geqslant n^{2^{m}-1}
$$

for all $n \geqslant K$. Note that the rightmost inequality of (3.2) follows since

$$
\left(\begin{array}{c}
n-m \\
2^{m}
\end{array}\right)
$$

is a polynomial in $\mathrm{n}$ of degree $2^{\mathrm{m}}$. As in the proof of Theorem 2 , we can then establish that eventually

$$
N(x) \geqslant(\log x)^{2^{m}-1}
$$


for any integer $m$. Therefore, for any integer $t$,

$$
N(x) \geqslant(\log x)^{t}
$$

eventually.

4. Ail 43YMPTCT CO FOR:UIAA FOR $\mathrm{N}_{\mathrm{k}}(\mathrm{x})$.

In what follows, for positive integers $k$ and $n$, we will denote the decimal representation of $x \in\left[0,10^{n}\right)$, where $\left[0,10^{n}\right)$ is the set of nor-negative integers less than $10^{\mathrm{n}}$, as

$$
\sum_{i=0}^{n-1} x_{i} 10^{i}
$$

Note that initial zeros will be allowed so that $\mathrm{x}$ will have $\mathrm{n}$ digits. For each $j=0,1,2, \ldots, e(k)-1$ we also define the finite sequences $B(x, j)$ and $T(x)$ by

$$
T(x)=\left\{x_{i}\right\}^{\bar{e}(k)-1}
$$

and

$$
B(x, j)=\{x \bar{e}(k)+j+i e(k)\}_{1=0}^{f}
$$

where

$$
f=\left[\frac{n-\bar{e}(k)-1}{e(k)}\right] \text {. }
$$

Using (4.2) and (4.3), we now define the relation $\cong$ on $\left[0,10^{n}\right.$ ) by: $x \cong y$ if and only if $T(x)=T(y)$ and $B(x, j)$ is a rearrangement of the terms of $B(y, j)$ for each $j$.

It is clear that $\cong$ is an equivalence relation on $\left[0,10^{n}\right)$. For $x$ a member of $\left[0,10^{n}\right)$, let $\langle x\rangle$ denote the equivalence class containing $x$. The following lemma will be used to help count the number of vivan numbers with dicital sum $r$.

innt 3. Tot $x, y \in\left[0,10^{n}\right)$. Then $x \cong y$ inn? that $a(y)=s(y$ :

ard $x \equiv y(n ; p)$.

FROC. Stince $x \cong y$, we have that $T(x)=T(y)$ and $3(x, j)$ is a rearrancement of the terms of $E(y, j)$ for each $j=0,1,2, \ldots, e(k)-1$. Thus

$$
\sum_{i=0}^{\bar{n}(k)-1} x_{i}=\sum_{i=0}^{\bar{e}(k)-1} y_{j}
$$

ar:

$\sum_{j=0}^{e(k)-1} \sum_{i=0}^{f(i)} x-(k)+j+i e(k)=\sum_{j=0}^{e(k)-1} \sum_{i=0}^{f(j)} \sqrt{e}(k)+j+j e(k)$ 
where for each $j$,

$$
f(j)=\left[\frac{n-\bar{e}(k)-1-j}{e(k)}\right]
$$

Wence, by adding corresponding sides of $(4.5)$ and $(4.6)$, we have that $s(x)=s(y)$. Note also that for each $j$,

$$
10^{\bar{e}(k)+j+r e(k)} \equiv 10^{\bar{e}(k)+j+t e(k)(\bmod k)}
$$

for any pair of non-negative integers $r$ and $t$. Thus, $x \equiv y$ (mod $k$ ).

LEMPA 4. Let $x \in\left[0,10^{n}\right)$. Then $x \in N_{k}$ if and only if $\langle x\rangle \subseteq N_{k}$. PROOF. Since $x \in\langle x\rangle$, it is immediate that $\langle x\rangle \subseteq N_{k}$ implies that

$x \in N_{k}$. Sorversely, suppose that $y \in\langle x\rangle$. Then $x \cong z$ and by Lemna 3 , $x=s(x)=s(y)$ and $y \equiv x \equiv 0($ mod $k)$. Therefore, $y \in N_{k}$ and we have that $\langle x\rangle \subseteq \mathrm{N}_{\mathrm{k}}$.

!ote that Lemma 4 states either an equivalence class contains only Niven numbers, or it contains only non-Niven numbers.

For a finite sequence

$$
\left\{a_{i}\right\}_{i=0}^{m}
$$

of digits, let

$$
d_{t}=\#\left\{i: a_{i}=t\right\} \text {, }
$$

for $t=0,1,2, \ldots, 9$. Here, the \# symbol denotes the cardinality of the set. For example, if $t=3$, then $d_{3}$ is the number of terms of the sequence equal to 3 . Therefore, the number of finite sequences which can be formed by rearranging the terms of $(4.9)$ is given by the multinomial coefficient

$$
\left(\begin{array}{c}
m+1 \\
d_{0}, d_{1}, \ldots, d_{9}
\end{array}\right)
$$

Ve will use this fact to develop an asymptotic formula for $\mathrm{N}_{k}(x)$ for any integer k.

I.GMMA 5. Let $x \in N_{k} \cap\left[0,10^{n}\right)$. Then $\#\langle x\rangle$ is a polynomial in $f$ of degree less than or equal to $k$ where

$$
f=\left[\frac{n-\bar{e}(k)-1}{e(k)}\right]
$$

PROOF. Note that each $y \in\langle x\rangle$ may be found by rearranging the terms of $B(x, j)$ for various $j^{\prime} s$. Let

$$
d_{t}(j)=\#\{0 \leqslant i \leqslant f: x-(k)+j+i e(k)=t\} \text {. }
$$

By the previous discussion, the number of such $y^{\prime} s$ which can be formed by these rearrangements is given by

$$
\prod_{j=0}^{e(k)-1}\left(\begin{array}{c}
f+1 \\
d_{0}(j), d_{1}(j), \ldots, d_{9}(j)
\end{array}\right) .
$$


But each factor of this product is a polynomial in $f$ of degree

$$
\sum_{i=1}^{9} d_{i}(j)
$$

and so, $\#\langle x\rangle$ is a polynomial in $f$ of degree

$$
\sum_{j=0}^{e(k)-1} \sum_{i=1}^{9} d_{i}(j)
$$

which is less than or equal to $k$.

THEOREM 6 . Let $n, k$ be positive integers. Then

$$
\mathrm{N}_{\mathrm{k}}\left(10^{\mathrm{n}}\right) \sim \mathrm{cn}^{\mathrm{k}}
$$

for a constant $c$ which depends upon $k$.

PROOF. Since

$$
N_{k}\left(10^{n}\right)=\sum \#\langle x\rangle \text {. }
$$

where the sum is taken over the collection of equivalence classes induced by $\cong$ on $\mathrm{N}_{k} \cap\left[0,10^{n}\right)$, we have that $\mathrm{N}_{k}\left(10^{n}\right)$ is a polynomial in $f$ of degree not, exceeding $k$ by Lemma 5. Thus, all we need to do in order to show that $N_{k}\left(10^{n}\right)$ has degree $k$ is to constmact a Niven number, $x$, with digital sum $k$ such that $\langle x\rangle$ is a polynomial in $f$ of degree $k$. Such an $x$ is

$$
\sum_{i=0}^{k-1} 10^{\bar{e}(k)+1 e(k)}
$$

Here,

$$
\#\langle x\rangle=\left(\begin{array}{l}
f \\
k
\end{array}\right),
$$

which is a polynomial in $f$ of degree exactly $k$. Thus,

$$
\mathrm{N}_{k}\left(10^{n}\right) \sim c_{1} f^{k}
$$

for some $c_{1}$ which is dependent on $k$. Since

$$
f=\left[\frac{n-\bar{e}(k)-1}{e(k)}\right],
$$

we have that $f \sim n / e(k)$ and therefore, $N_{k}\left(10^{n}\right) \sim \mathrm{cn}^{k}$ where $0=c_{1} /(e(k))^{k}$. Finally, using an argument similiar to that in the proof of Theorem 2 , we have the following corollary.

COROLLARY 7. Let $k, x$ be positive integers. Then

$$
\mathrm{N}_{\mathrm{k}}(\mathrm{x}) \sim \mathrm{c}(\log \mathrm{x})^{\mathrm{k}}
$$

where $c$ depends on $k$. 
5. CONCLUSION.

Thus, a partial answer concerning an asymptotic formula for $N(x)$ has been presented. As was shown by Theorem 2 , exact values of the constant $c$ can be found for certain integers $k$. In fact, given a particular $k$, it is indeed possible to determine the exact form that $c$ will be. This would involve an investigation of the partitions of $k$ with summands less than or equal to 9 , and the number of solutions to certain diophantine congruences. We feel that this is a subject for future stury. The determination of an asymptotic formula for $:(x)$, however, will he left, an an open problem. REF?RANCES

1. KENNEDY, P. .., COCDNAN, T.A., BIST, C.H. Mathematical Discovery and Niven Numbers, The MATYC Journal 1.4 (1980), 21-25.

$\because$ KNNEIY, R. .., TOOPER, S.N. on the Natural Density of the Niven Numbers, Coilege Mathematies Joumal 15 (1984), 309-31?.

3. TANG, 3. . An Improvernent and Generalization of Bellman-Shapiro's Theorem on a Problem in Additive Number Theory, Froc. Amer. Math. Soc. 14 (1963), $199-204$. 




Advances in

Operations Research

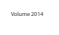

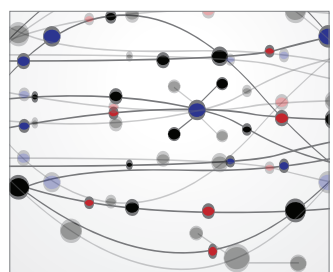

\section{The Scientific} World Journal
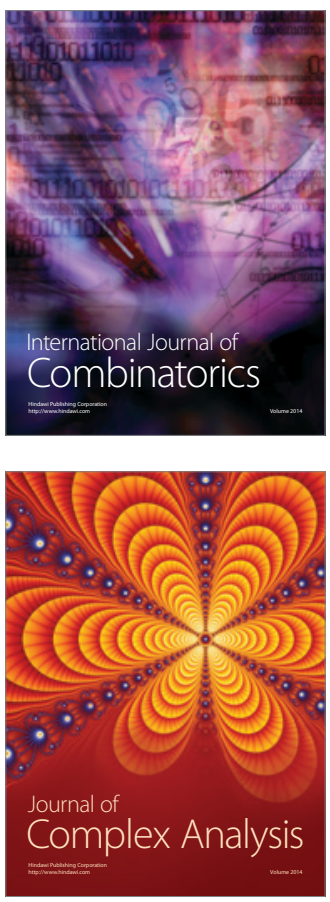

International Journal of

Mathematics and

Mathematical

Sciences
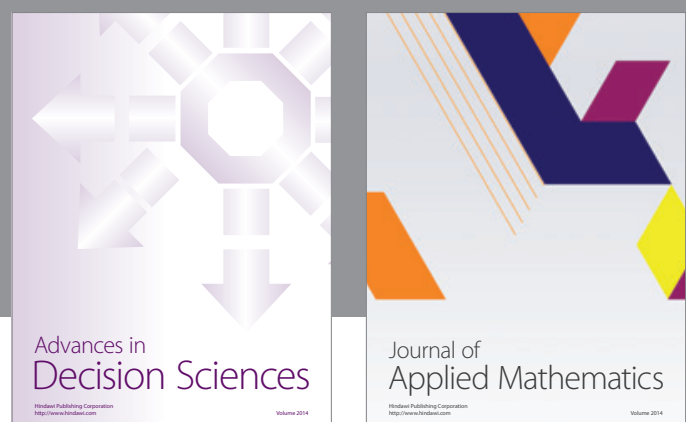

Journal of

Applied Mathematics
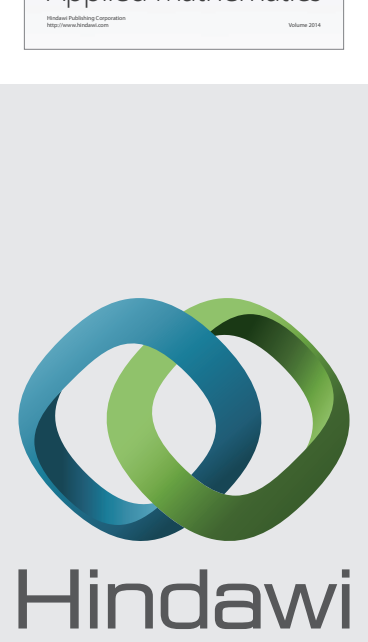

Submit your manuscripts at http://www.hindawi.com
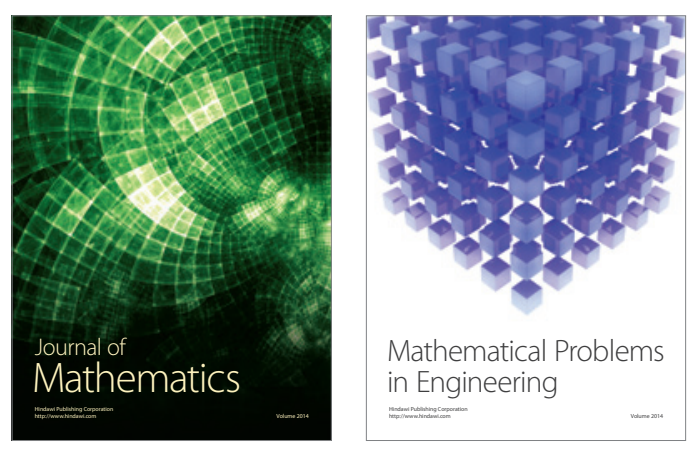

Mathematical Problems in Engineering
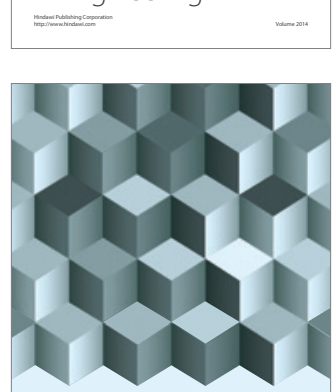

Journal of

Function Spaces
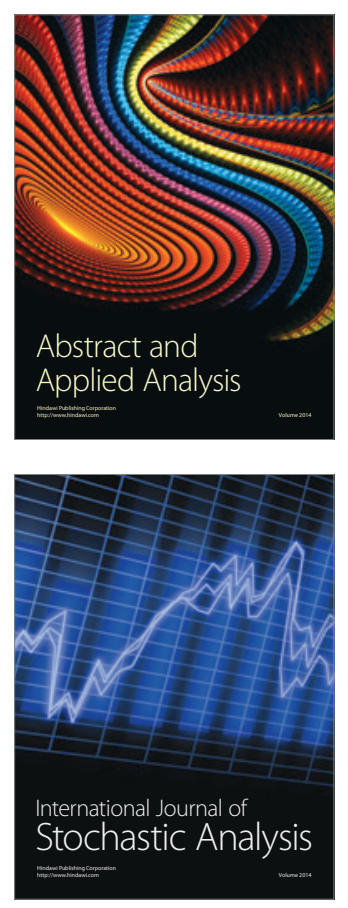



ournal of

Probability and Statistics

Promensencen
